\title{
Failure to show decrease in small pulmonary blood vessels in rats with experimental pulmonary hypertension
}

\author{
JM KAY, KL SUYAMA, PM KEANE \\ From the Department of Laboratory Medicine, St Joseph's Hospital, and Department of Pathology, Faculty of Health \\ Sciences, McMaster University, Hamilton, Ontario, Canada
}

ABSTRACT We induced chronic pulmonary hypertension in one group of rats by exposing them to chronic hypobaric hypoxia (380 $\mathrm{mm} \mathrm{Hg}$ for three weeks) and in another group by administering a single subcutaneous dose of monocrotaline $(60 \mathrm{mg} / \mathrm{kg}$ body weight $)$. Both groups of rats showed increase of the right ventricular mean systolic blood pressure and right ventricular hypertrophy. We measured the surface area of histological sections of the left or right lungs and counted all small blood vessels with an external diameter of less than $50 \mu \mathrm{m}$ and with a definite elastic coat lying distal to respiratory bronchioles. In the 10 rats with chronic hypoxic pulmonary hypertension the mean total number of small pulmonary blood vessels was $428.8 \pm 96.9$ (SD) compared with $337 \cdot 8 \pm 91.9$ in 10 untreated control rats. The number of small pulmonary blood vessels per $\mathrm{mm}^{2}$ of lung tissue was $4 \cdot 0 \pm 1 \cdot 3$ in the chronically hypoxic rats compared with $3.8 \pm 1.2$ in the controls. The mean total number of small pulmonary blood vessels in nine rats with monocrotaline-induced pulmonary hypertension was $396 \cdot 8 \pm 61 \cdot 7$ compared with $384 \pm 55 \cdot 4$ in three control rats. The number of small pulmonary blood vessels per $\mathrm{mm}^{2}$ lung tissue was $3.3 \pm 0.6$ in the rats treated with monocrotaline compared with $3 \cdot 6 \pm 0 \cdot 6$ in the control group. We conclude that the number of small pulmonary blood vessels is not reduced in rats with pulmonary hypertension induced by chronic hypoxia or monocrotaline.

Chronic pulmonary arterial hypertension can be induced in rats either by inducing chronic hypoxia' or by administering the pyrrolizidine alkaloid monocrotaline. ${ }^{2}$ In both these experimental models the pulmonary hypertension is accompanied by muscularisation of pulmonary arterioles and right ventricular hypertrophy. In the case of monocrotaline pulmonary hypertension, the muscular pulmonary arteries develop medial hypertrophy and sometimes show necrotising arteritis. It was suggested by one group of workers that a factor in the development of increased pulmonary vascular resistance in both monocrotaline pulmonary hypertension ${ }^{3}$ and chronic hypoxic pulmonary hypertension ${ }^{4}$ is a reduction in the number of small pulmonary arteries. This suggestion was based on visual assessment of postmortem angiograms, measurements of arterial number per unit area, and estimates of the ratio of arterial to alveolar number in histological sections. This view has been challenged, however, by another group of workers, who used labelled albumin to measure the pulmonary vascular volume in rats. ${ }^{5}$ They found that the total vascular volume of the left lung was not significantly different in

Reprint requests to: Dr. JM Kay. Department of Laboratory Medicine, St Joseph's Hospital. Hamilton. Ontario L8N 1Y4. Canada. chronically hypoxic and control groups of rats, despite lower body weight in the former group. Recently we have studied the development of pulmonary hypertension, pulmonary vascular disease, and right ventricular hypertrophy in rats exposed to chronic hypobaric hypoxia ${ }^{67}$ and rats given a single subcutaneous injection of monocrotaline. ${ }^{8}$ In these experiments all the small pulmonary blood vessels measuring less than $50 \mu \mathrm{m}$ in diameter were counted in histological sections of either the left or the right lung. Since it is important to know whether there is destruction of the pulmonary vascular bed in pulmonary hypertension, we have re-examined the data from our experiments to determine whether or not there was a reduction in small pulmonary blood vessels in rats subjected to chronic hypoxia or treated with monocrotaline.

\section{Methods}

CHRONIC HYPOXIC PULMONARY HYPERTENSION Twenty female Wistar rats were divided into control and test groups of equal number. The initial mean body weights of the two groups $71 \cdot 2 \pm 6 \cdot 6 \mathrm{~g}(\mathrm{SD})$ and $75 \cdot 7 \pm$ $13 \cdot 2 \mathrm{~g}$. The 10 test rats were confined in a cylindrical steel hypobaric chamber, which was initially decompressed to 
$460 \mathrm{~mm} \mathrm{Hg}$. The pressure was progressively reduced in daily stages so that after one week it was $380 \mathrm{~mm} \mathrm{Hg}$. The rats remained at this pressure for a further two weeks. After three weeks of chronic hypoxia, the right ventricular mean systolic blood pressure (Prvs) was measured in the control and test rats by cannulating the right external jugular vein under light ether anaesthesia. The rats were then killed and the thoracic organs removed. The trachea was cannulated and the lungs were distended with $13 \mathrm{ml}$ of $10 \%$ formol saline, which ensured that the pleural surfaces were smooth. The trachea was then ligated, the cannula withdrawn and the viscera immersed in $10 \%$ formol saline until fixation was complete. The hearts were dissected and right ventricular hypertrophy was evaluated by expressing the weight of the free wall of the right ventricle as a percentage of the weight of the left ventricle and interventricular septum. A single block of the entire left lung cut in the parasagittal plane was routinely processed for histological examination. Sections were stained by the Miller elastic-Van Gieson method ${ }^{9}$ for distinguishing smooth muscle, collagen, and elastic tissue. The small pulmonary blood vessels were evaluated by the method described below.

MONOCROTALINE-IN DUCED PULMONARY

HYPERTENSION

We studied a group of nine test rats and a group of three control rats whose respective initial mean body weights were $123.7 \mathrm{~g} \pm 5.8$ and $108.3 \mathrm{~g} \pm 11.9$. Each test rat was given a single subcutaneous injection of a $2 \%$ aqueous solution of monocrotaline hydrochloride. The dose of monocrotaline was $60 \mathrm{mg} / \mathrm{kg}$ body weight. On the 14th, 17 th, and 22nd days after the injection of monocrotaline the Prvs was measured in one control and three test rats. The rats were then killed and the cervical and thoracic organs removed in one block. The left main bronchus was clamped and ligated, after which the left lung was removed for enzyme assay. ${ }^{8}$ The trachea was cannulated and the right lung was distended by injecting $7 \mathrm{ml}$ of $10 \%$ formol saline, which ensured that the pleural surfaces were smooth. The trachea was then ligated, the cannula withdrawn and the remaining thoracic viscera immersed in $10 \%$ formol saline until fixation was complete. The hearts were dissected and right ventricular hypertrophy was evaluated as described above. A block of tissue was cut from each of the superior, middle, inferior, and median lobes of the right lung of every animal and was routinely processed for histological examination. Sections stained by the Miller elastic-Van Gieson method were used for assessment of the small pulmonary blood vessels as described below.

EVALUATION OF SMALLPULMONARY BLOOD VESSELS

The muscular pulmonary artery in a rat is defined as an arterial vessel with an external diameter of between 20 and $400 \mu \mathrm{m}$. It has a tunica media of circularly orientedo smooth muscle sandwiched between two elastic laminae. The pulmonary arteriole in a rat is an arterial vessel witho an external diameter of under $20 \mu \mathrm{m}$ whose wall is $\overline{\mathrm{D}}$ normally devoid of smooth muscle except adjacent to itso origin from a muscular pulmonary artery. It consists of as single elastic lamina lined by endothelium. Pulmonary. venules are structurally identical to pulmonary arterioles $\overrightarrow{\vec{\omega}}$ and these cannot be distinguished from one another unlesso they can be traced to a larger vessel. We used the technique of Hunter and coworkers to assess the small pulmonary blood vessels. ${ }^{10}$ The histological sections of. lung were systematically examined with the $\times 40 \vec{N}$ objective in a microscope fitted with a calibrated eyepiece micrometer. All small blood vessels less than $50 \mu \mathrm{m} v$ external diameter with a definite elastic coat lying distal to응 respiratory bronchioles were counted. These vessels lie $\overrightarrow{-}$ adjacent to alveolar ducts or alveolar spaces. The countsक included small muscular pulmonary arteries, normali్ pulmonary arterioles, and, in the case of rats with? pulmonary hypertension, muscularised pulmonary arter-은 ioles. The counts of vessels also included occasional small $\vec{\bullet}$ venules, which could not be distinguished from normal pulmonary arterioles. After the vessels had been counted the histological sections were inserted into a Kodako Carousel 750 slide projector and images were projected on to paper at a distance of $3 \mathrm{~m}$. The sections of lung were⿳亠丷厂 outlined on the paper with a pencil. The images were then cut out and weighed using a Sartorius semimicro analytical balance. The area of each lung section wasō calculated by comparing the weight of the paper images 3 with the weight of a square centimetre projected on to identical paper at the same distance. All sections were coded and examined without knowledge of whether they were derived from control or test rats.

The results of the two experiments expressed as means: with SD were evaluated with the $t$ test for unpaired data. 3 Differences in mean values were considered to be significant when $p<0.05$.

\section{Results}

In the table are shown the initial and final body weights, $\bar{P}$ rvs, right ventricular weight, number of small pulmon- $\tilde{\sigma}$ ary blood vessels, and area of lung sections in control rats $N$ and rats with pulmonary hypertension due to chronic N hypoxia or to monocrotaline. The groups of rats exposed to chronic hypoxia and given monocrotaline both had pulmonary hypertension associated with right ventricular? hypertrophy. Both types of treatment interfered with normal growth and so the final body weights of the two groups of test rats were lower than their respective contrologroups. Despite this reduction in body weight, the area of the lung sections was increased in both the chronically? hypoxic rats and those treated with monocrotaline. The total number of small pulmonary blood vessels was< 
Effect of chronic hypoxia and monocrotaline on right ventricular mean systolic blood pressure, right ventricular weight, number of small pulmonary blood vessels, and area of lung sections in rats.

\begin{tabular}{|c|c|c|c|c|}
\hline & \multicolumn{2}{|c|}{ Chronic hypoxia experiment } & \multicolumn{2}{|c|}{ Monocrotaline experiment } \\
\hline & Controlt & Test' & Control $(n=3)$ & Test $1 \div$ \\
\hline Initial body weight (g) & $71 \cdot 2 \pm 6.6$ & $75 \cdot 7 \pm 13 \cdot 2$ & $108 \cdot 3 \pm 11 \cdot 9$ & $123 \cdot 7 \pm 5 \cdot 8$ \\
\hline Final body weight (g) & $185.6 \pm 15 \cdot 1$ & $157 \cdot 8 \pm 22.9 *$ & $197 \cdot 3 \pm 22 \cdot 2$ & $183.8 \pm 12 \cdot 6$ \\
\hline Prvs $(\mathrm{mm} \mathrm{Hg})$ & $\begin{array}{c}19 \cdot 6 \pm 3.5 \\
(n=6)\end{array}$ & $\begin{array}{c}37 \cdot 3 \pm 6 \cdot 4 * \\
(n=9)\end{array}$ & $24.7 \pm 6.6$ & $\begin{array}{c}50 \cdot 5 \pm 11 \cdot 3 * \\
(n=8)\end{array}$ \\
\hline $\mathrm{RV} /(\mathrm{LV}+\mathrm{S}) \%$ & $28 \cdot 8 \pm 3 \cdot 6$ & $48 \cdot 5 \pm 7 \cdot 7 *$ & $30 \cdot 3 \pm 4 \cdot 1$ & $48 \cdot 5 \pm 10 \cdot 0^{*}$ \\
\hline Total SPBV & $337.8 \pm 91.9$ & $428.8 \pm 96.9 *$ & $384 \cdot 0 \pm 55 \cdot 4$ & $396.8 \pm 61.7$ \\
\hline Area of lung section $\left(\mathrm{mm}^{2}\right)$ & $90 \cdot 7 \pm 18 \cdot 1$ & $107.2 \pm 19.4$ & $95 \cdot 6 \pm 5.2$ & $121 \cdot 6 \pm 12 \cdot 0 *$ \\
\hline $\mathrm{SPBV} / \mathrm{mm}^{2}$ & $3.8 \pm 1.2$ & $4.0 \pm 1.3$ & $3.6 \pm 0.6$ & $3 \cdot 3 \pm 0.6$ \\
\hline
\end{tabular}

*Test result significantly different from control. $\mathrm{p}<0 \cdot 05$.

$\dagger n=10$ except where otherwise stated. $\div n=9$ except where otherwise stated.

Prvs-right ventricular mean systolic blood pressure: $R V$ - weight of free wall of right ventricle: $L V+S-$ weight of left ventricle and interventricular septum: SPBV - small pulmonary blood vessels

significantly increased in the chronically hypoxic rats. When this number was expressed per unit area of lung tissue, however, there was no significant difference between test and control groups. In the rats with monocrotaline-induced pulmonary hypertension the total number of small pulmonary blood vessels was increased but the difference between test and control values did not achieve significance. Similarly, there was no significant difference between the monocrotaline test and control groups when the number of small pulmonary blood vessels was expressed per unit area of lung tissue.

\section{Discussion}

The first report of a reduction in pulmonary arteries in monocrotaline pulmonary hypertension was published by Hislop and Reid in $1974 .{ }^{3}$ These workers fed male Sprague-Dawley rats weighing 170-192 g a diet containing powdered Crotalaria spectabilis seeds. These seeds contain the pyrrolizidine alkaloid monocrotaline. After 34 days the test rats showed right ventricular hypertrophy and an increase in the medial thickness of muscular pulmonary arteries. The number of small pulmonary arteries was reduced from 8.51 per $\mathrm{mm}^{2}$ in control rats to 4.63 per $\mathrm{mm}^{2}$ in rats fed Crotalaria spectabilis seeds. The ratio of the number of alveoli to the number of arteries increased from 26.61 in control animals to 49.98 in test rats. In 1976 the same group of workers exposed male Sprague-Dawley rats weighing 175-225 g to hypobaric hypoxia ( $380 \mathrm{~mm} \mathrm{Hg}$ ) for three to 28 days. ${ }^{4}$ After 14 days' exposure the number of pulmonary arteries measuring less than $200 \mu \mathrm{m}$ diameter was reduced by $40 \%$.

We are unable to confirm the results of Hislop and Reid with regard to either monocrotaline-induced pulmonary hypertension or chronic hypoxic pulmonary hypertension. Rather our findings support the work of Hunter $e t$ $a l,{ }^{10}$ who found no reduction in the number of small arteries in male Wistar rats living in a decompression chamber at $390 \mathrm{~mm} \mathrm{Hg}$. In a similar study Leach (quoted by Emery $e \mathrm{al}^{5}$ ) counted all small vessels less than $50 \mu \mathrm{m}$ in diameter in the left lung of rats and found no evidence of a loss of vessels in animals exposed to $8 \%$ or $10 \%$ oxygen. Specifically, the mean number of vessels was 342 $\pm 40(\mathrm{SEM})$ in 50 control rats and $332 \pm 12$ in 50 chronically hypoxic rats. Recently Emery and her colleagues ${ }^{5}$ investigated this problem using a different method. They perfused the lungs of control rats and chronically hypoxic rats kept in $10 \%$ oxygen for three to six weeks with a solution containing human serum albumin labelled with radioactive iodine. The vascular volume of the left lung was then calculated from measurements of gamma emissions. The method was sensitive enough to detect changes in vascular volume with growth. They found that the vascular volume of the lung in the chronically hypoxic rats was not reduced. In fact, it was large for the body size and normal for the age of the rats.

When pulmonary blood vessels become obliterated as a result of fibrosing diseases of the lung " or thrombotic occlusion, their previous existence can be inferred by recognition of nodules of fibroelastic tissue. We have never seen such evidence of vascular obliteration in rats with pulmonary hypertension due to chronic hypoxia or monocrotaline. Hislop and Reid saw no sign of "lost" vessels in their rats with pulmonary hypertension due to chronic hypoxia despite a $40 \%$ reduction in the number of pulmonary arteries. ${ }^{4}$ In their rats fed Crotarlaria spectabilis seeds they described non-muscular, partially muscular, and muscular "ghost" arteries in which the lumen was reduced or blocked by enlarged intimal cells. ${ }^{3}$ They speculated that such blocking led to obliteration and disappearance of the arteries.

Presumably the discrepancy between the results of Hislop and Reid ${ }^{34}$ on the one hand and Hunter, ${ }^{10}$ Leach, Emery, ${ }^{5}$ and ourselves on the other hand is related to differences in the technique of preparing the lungs for examination and the enumeration of pulmonary blood vessels. Hislop and Reid removed the thoracic viscera in one block and deep-froze them. Later the lungs were thawed and the pulmonary arteries injected with a mixture of barium sulphate and gelatin at a temperature of $60^{\circ} \mathrm{C}$ and a pressure of $100 \mathrm{~cm} \mathrm{H}_{2} 0$. The lungs were 
distended through the trachea with $10 \%$ buffered formol saline until the pleural surfaces were smooth. The numbers of small arteries and alveoli in a given area of tissue were counted. The size of the given area is not stated but in more recent papers about chronic hypoxia ${ }^{12}$ and Crotalaria spectabilis, ${ }^{1314}$ Reid and her colleagues have counted alveoli and blood vessels in 15, 20, or 25 microscopic high-power fields. In both chronic hypoxia ${ }^{10}$ and monocrotaline intoxication the lungs are enlarged. Thus if the total number of vessels remains roughly constant, observations on a limited area of the lung sections instead of the entire area would give an impression of a reduction in vessel number. It is important that this matter is clarified since it has pathogenetic and prognostic implications for human pulmonary vascular disease. Specifically Reid and her colleagues have suggested on the basis of similar counting methods that there is a reduction in the number of pulmonary arteries in primary pulmonary hypertension ${ }^{15}$ and congenital heart lesions such as ventricular septal defect, atrioventricular defect, and complete transposition of the great arteries. ${ }^{16}$

We thank Jennifer Hines for assistance in the preparation of the manuscript. The work was supported by grants from the Medical Research Council of Canada and St Joseph's Hospital Foundation.

\section{References}

${ }^{1}$ Abraham AS, Kay JM, Cole RB, Pincock AC. Haemodynamic and pathological study of the effect of chronic hypoxia and subsequent recovery of the heart and pulmonary vasculature of the rat. Cardiovasc Res 1971;5:95-102.

${ }^{2}$ Kay JM, Heath D. Crotalaria spectabilis: the pulmonary hypertension plant. Springfield: Charles C Thomas, 1969.

${ }^{3}$ Hislop A, Reid L. Arterial changes in Crotalaria spectabilisinduced pulmonary hypertension in rats. BrJ Exp Pathol 1974;55:153-63.
${ }^{4}$ Hislop A, Reid L. New findings in pulmonary arteries of rats with hypoxia-induced pulmonary hypertension. $B r J$ Exp Pathol 1976;57:542-54.

${ }^{5}$ Emery CJ, Bee D. Barer GR. Mechanical properties and reactivity of vessels in isolated perfused lungs of chronically hypoxic rats. Clin Sci 1981;61:569-80.

${ }^{6}$ Kay JM. Effect of intermittent normoxia on chronic hypoxic pulmonary hypertension, right ventricular hypertrophy, and polycythemia in rats. Am Rev Respir Dis 1980;121:9931001.

${ }^{7}$ Kay JM, Suyama KL, Keane PM. Effect of intermittent normoxia on muscularisation of pulmonary arterioles induced by chronic hypoxia in rats. Am Rev Respir Dis 1981;123:454-8.

${ }^{8}$ Kay JM, Keane PM, Suyama KL, Gauthier D. Angiotensin converting enzyme activity and evolution of pulmonary vascular disease in rats with monocrotaline pulmonary hypertension. Thorax 1982;37:88-96.

${ }^{9}$ Miller PJ. An elastin stain. Med Lab Technol 1971;28:148-9.

${ }^{10}$ Hunter C. Barer GR, Shaw JW, Clegg EJ. Growth of the heart and lungs in hypoxic rodents: a model of human hypoxic disease. Clin Sci Mol Med 1974;46:375-91.

" Heath D, Gillund TD, Kay JM, Hawkins CF. Pulmonary vascular disease in honeycomb lung. $J$ Pathol Bacteriol 1968;95:423-30.

12 Rabinovitch M, Gamble W, Nadas AS, Miettinen OS, Reid L. Rat pulmonary circulation after chronic hypoxia:hemodynamic and structural features. Am J Physiol 1979;236:H818-27.

${ }^{13}$ Meyrick B, Reid L. Development of pulmonary arterial changes in rats fed Crotalaria spectabilis. Am J Pathol 1979;94:37-50.

${ }^{14}$ Meyrick B, Gamble W, Reid L. Development of Crotalaria pulmonary hypertension: hemodynamic and structural study. Am J Physiol 1980;239:H692-702.

${ }^{15}$ Anderson EG, Simon G, Reid L. Primary and thromboembolic pulmonary hypertension: a quantitative pathological study. J Pathol 1973;110:273-93.

${ }^{16}$ Rabinovitch M, Haworth SG, Vance Z, et al. Early pulmonary vascular changes in congenital heart disease studied in biopsy tissue. Hum Pathol 1980;11:499-509. 